\title{
THE UNAVOIDABLE ERROR IN THE DIFFERENTIAL COUNT OF THE LEUKOCYTES OF THE BLOOD
}

\author{
By C. W. BARNETT \\ (From the Department of Medicine, Stanford University Medical School, San Francisco)
}

(Received for publication August 8, 1932)

In differential blood counting, one attempts by careful observation of a limited number of leukocytes to obtain a picture of the actual distribution of the various types of cells in the blood stream. Many physicians are inclined to accept a count done by a competent person as a very close approximation to this value. There are many statements in the literature which indicate an absolute confidence in the results of differential counts, of which the following quotation from an article on surgical lesions of the abdomen is a typical example. "Probably the blood count is of the greatest value in determining the severity of the lesion in acute appendicitis. When the polymorphonuclear percentage is 70 , or below, a suppurative or gangrenous process may be ruled out and operation delayed or postponed with safety. It is difficult to be sure of the severity of the process when the percentage of polymorphonuclears is between 70 and 80 . I have seen beginning peritonitis with percentages below 80 but above this point one may feel sure that he is dealing with a dangerous lesion which should have immediate treatment."

Such confidence is unwarranted, since differential counting of the leukocytes is one of the most uncertain of the quantitative methods used in medicine. There are three types of error in this procedure. The first is the mechanical error, which includes all variations in taking the blood sample, making the smear and staining, and all irregularities in distribution of the cells depending upon the type of smear and the areas over which the count is made. The second type is the error of interpretation, the magnitude of which depends entirely upon the judgment of the observer. The third is the error due entirely to chance, which is the subject of the present paper.

Many articles have been written on the errors of blood counting, but most of them are concerned with the mechanical errors. Thus, Napier (1), Meissner (2), Stephens et al. (3), Beacom (4) and Gyllenswärd (5) describe variations in differential counts on smears made in various ways, the counts being made over various areas of the smears. No two of these authors agree as to the most accurate method of making the count. The chance errors either are not mentioned or are discussed very briefly. All 
agree, however, that at least 300 cells must be counted if the results are to be at all accurate.

Dahlberg (6) attempts to determine the magnitude of the various types of error and gives variabilities \pm 4.3 per cent for the mechanical error, \pm 11 per cent for the error of interpretation and \pm 5.8 per cent for the chance error on counts of 500 cells. The data used as a basis for these results are not given, and the exact meaning of the results is not clear. Kilgore (7) calculated the chance error on a count of 100 cells and found that with an actual count of 50 per cent lymphocytes, different observations on the same blood would be expected to vary between 35 per cent and 65 per cent. He did not check the calculation with any actual counts.

Brandt (8) studied the mechanical and chance errors in red, total white and differential white blood counts. He calculated that in counting 500 cells with 66 per cent of polymorphonuclears, the standard deviation would be 2.11 per cent of the total count or 3.2 per cent of 66 . He compared this with six counts of 500 cells each in which he obtained a standard deviation of only 1.4 per cent of 66 . Six, however, is far too small a number of counts for an accurate determination of the standard deviation.

An error due entirely to chance occurs whenever in dealing with a universe composed of a very large number of individual units our estimate of the distribution of different types of units must be based upon the study of an extremely small proportion of them. Thus in differential counting we study a few hundred cells in order to determine the proportion of the various types in the entire blood stream, which, if we assume a white count of 10,000 cells per cubic millimeter, and a blood volume of 5 liters, will contain $50,000,000,000$ cells. It is obvious that when from such a tremendous total number of cells, samples as small as one hundred are taken, each will differ slightly from another, and no single count will give a true picture of the actual distribution. This error is completely independent of technique or interpretation and is entirely unavoidable. It can be decreased by increasing the number of cells studied in the total count, but it cannot be eliminated, and when small numbers of cells are counted, it leads to a large variation as will be shown.

Even when the same blood is subjected to a series of counts, there will be a certain variation or dispersion in the results. The simplest method of expressing the magnitude of this dispersion is to note the range between the highest and lowest values obtained for a particular type of cell. This method is misleading, however, because a single count that happens to be far above or below the others will be unduly weighted. The commonest and most satisfactory expression of the magnitude of the dispersion is obtained by dividing the sum of the squares of the differences between the individual observations and the mean value by the total 
number of observations and taking the square root of the result. This measure of dispersion is called the standard deviation.

If the standard deviation can be determined, it is easy to estimate the amount of variation to be expected as the result of chance alone. The chances are approximately 2 to 1 that a particular observation will fall within the bounds of one standard deviation above or below the actual value, about 20 to 1 that it will fall within twice the standard deviation, and about 370 to 1 that it will fall within three times the standard deviation above or below the true value. It is generally considered that chance errors may be as great as three times the standard deviation above or below the actual value.

In differential blood counts, the standard deviation can be determined readily according to the formula, from Yule (9), page 257:

$$
\begin{aligned}
& \qquad \sigma=\sqrt{N p q .} \\
& \sigma=\text { the standard deviation, } \\
& N=\text { the number of cells counted, } \\
& p=\text { the proportion of cells of a certain type, } \\
& q=\text { the proportion of all other types of cells. }
\end{aligned}
$$

This formula gives the standard deviation in per cent of the total count. Thus if we count 100 cells with 25 per cent of lymphocytes and wish to find the standard deviation, we will have

$$
\begin{aligned}
N & =100, \\
p & =.25, \\
q & =.75, \\
\sigma & =\sqrt{100 \times .25 \times .75}=4.3 .
\end{aligned}
$$

We would expect chance errors to give a variation of three times this value above and below the actual value of 25 per cent. Three times 4.3 is 12.9 ; consequently a single count on 100 cells might give a lymphocyte percentage anywhere from 12.1 per cent to 37.9 per cent. Similar calculations will give the expected variations at all percentages from 0 to 100.

This chance variation is not entirely theoretical, and it can be checked by actual counts. For this purpose, 100 differential counts were done on the same blood. A sample of blood was taken with a little sodium citrate to prevent immediate coagulation, and fifty smears were made by the cover glass method as rapidly as possible. These were stained, and those with eight or more satisfactory low power fields were selected. On each smear, six counts of 100 cells each were made consecutively, all the counts being done in areas where the red cells were just separated from each other. Since all the smears were made with the same blood and in the same way, it was hoped that the mechanical error would be eliminated or 
would be of about the same magnitude in each count. All the counts were made by one individual, which should make the error of interpretation negligible. The observed variations should therefore be almost entirely the result of chance.

The present work is concerned only with the chance distribution of observations about certain mean percentages, and consequently the actual type of cell in any group is of no significance. Therefore, any two or more types may be taken together to form a single larger group, providing the combination is made in each individual count. Several such groups were made in order to increase the range of observations from 50 per cent to 100 per cent. To avoid repetition of the names of the different cell types, they will be designated by letters as follows:

$$
\begin{aligned}
a & =\text { basophiles, } \\
b & =\text { eosinophiles, } \\
c & =\text { large mononuclears, } \\
d & =\text { lymphocytes, } \\
e & =\text { polymorphonuclears, } \\
g & =\text { polymorphonuclears + lymphocytes, } \\
h & =\text { polymorphonuclears + lymphocytes + large mononuclears, } \\
i & =\text { polymorphonuclears + lymphocytes + large mononuclears } \\
& \quad+\text { eosinophiles. }
\end{aligned}
$$

Occasional atypical cells were found that could not be placed in any of the above classes. The number of these was noted to preserve the true percentages of the other types, but they were not included in the calculation.

When the counts were completed, frequency tables were prepared for each type of cell. For all types except $a, b$, and $i$, the dispersion was large enough so that the listing of each individual percentage in which an observation happened to fall would make a table that contained too many classes. Therefore, the percentages were grouped in pairs, with a class interval of 2 per cent, and the number of observations that fell into each class was counted and listed in a frequency table under $f$. The means and standard deviations were calculated from the tables in the usual way (cf. Yule, pp. 110 and 134).

Table $I$ is a frequency table for cells of type $e$. It is given in full to indicate the method of calculation. Table II is a summary of the means and the theoretical and observed standard deviations for each type of cell.

Examination of the tables shows that in every case there is a close agreement between the standard deviation calculated for the percentage of cells in question and the actual standard deviation of the observed values. In other words, the dispersion observed in a series of 100 differential counts was just what was to be expected from calculation of the theoretical dispersion. In nearly every case, the observed standard 
deviation was slightly higher than the theoretical one. This is obviously due to the fact that the complete elimination of mechanical errors was impossible, but the close agreement demonstrates that they were reduced to a very small value.

TABLE I

Frequency table for cells of type $e$

\begin{tabular}{|c|c|c|c|c|}
\hline Class & $f$ & $x$ & $f x$ & $f x^{2}$ \\
\hline \multirow[t]{2}{*}{ 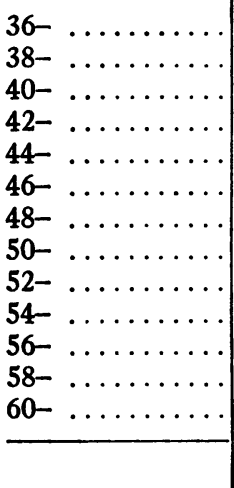 } & $\begin{array}{r}1 \\
1 \\
1 \\
7 \\
5 \\
10 \\
16 \\
15 \\
18 \\
12 \\
4 \\
6 \\
4\end{array}$ & $\begin{array}{r}-14 \\
-12 \\
-10 \\
-8 \\
-6 \\
-4 \\
-2 \\
0 \\
+2 \\
+4 \\
+6 \\
+8 \\
+10\end{array}$ & $\begin{array}{r}-14 \\
-12 \\
-10 \\
-56 \\
-30 \\
-40 \\
-32 \\
0 \\
+36 \\
+48 \\
+24 \\
+48 \\
+40\end{array}$ & $\begin{array}{r}196 \\
144 \\
100 \\
448 \\
180 \\
160 \\
64 \\
0 \\
72 \\
192 \\
144 \\
384 \\
400\end{array}$ \\
\hline & 100 & & +2 & 2484 \\
\hline
\end{tabular}

$M=50.5+2 / 100=50.52$.

$\Sigma f x^{2} / N=2484 / 100=24.84$.

$\sigma^{2}=24.84-. \overline{02}^{2}=24.84$.

$\sigma=4.98$.

$\sigma_{1}=\sqrt{100 \times .51 \times .49}=5.0$.

Theoretical standard deviation $=5.0$.

Observed standard deviation $=4.98$.

TABLE II

Table showing the means and standard deviations of the different types of cells

\begin{tabular}{|c|c|c|c|c|}
\hline Type of cell & Mean $M$ & $\begin{array}{c}\text { Range of } \\
\text { observed values }\end{array}$ & $\begin{array}{c}\text { Observed standard } \\
\text { deviation }\end{array}$ & $\begin{array}{c}\text { Theoretical standard } \\
\text { deviation }\end{array}$ \\
\hline 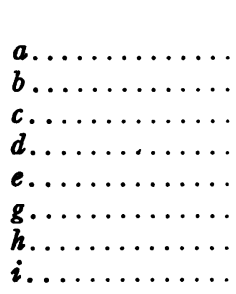 & $\begin{array}{r}0.52 \\
2.59 \\
16.62 \\
28.02 \\
50.52 \\
78.50 \\
94.42 \\
96.58\end{array}$ & $\begin{array}{l}0-3 \\
0-8 \\
7-27 \\
14-38 \\
37-60 \\
63-87 \\
87-99 \\
91-100\end{array}$ & $\begin{array}{c}\sigma \\
0.69 \\
1.67 \\
4.22 \\
5.05 \\
4.98 \\
4.59 \\
2.64 \\
2.03\end{array}$ & $\begin{array}{c}\sigma_{1} \\
0.71 \\
1.59 \\
3.67 \\
4.49 \\
5.00 \\
4.14 \\
2.37 \\
1.81\end{array}$ \\
\hline
\end{tabular}

All figures in this table are expressed in per cent of the total count.

In Chart 1, the individual observations on the percentages of groups $d, e$, and $g$ have been plotted to show the distribution of the counts in relation to multiples of the standard deviation. The actual percentage 
on any one count is obtained from the chart by adding to the mean value for the particular type of cell the deviation as measured on the vertical axis. The mean value is indicated on the horizontal axis by a vertical line drawn through the group of spots.

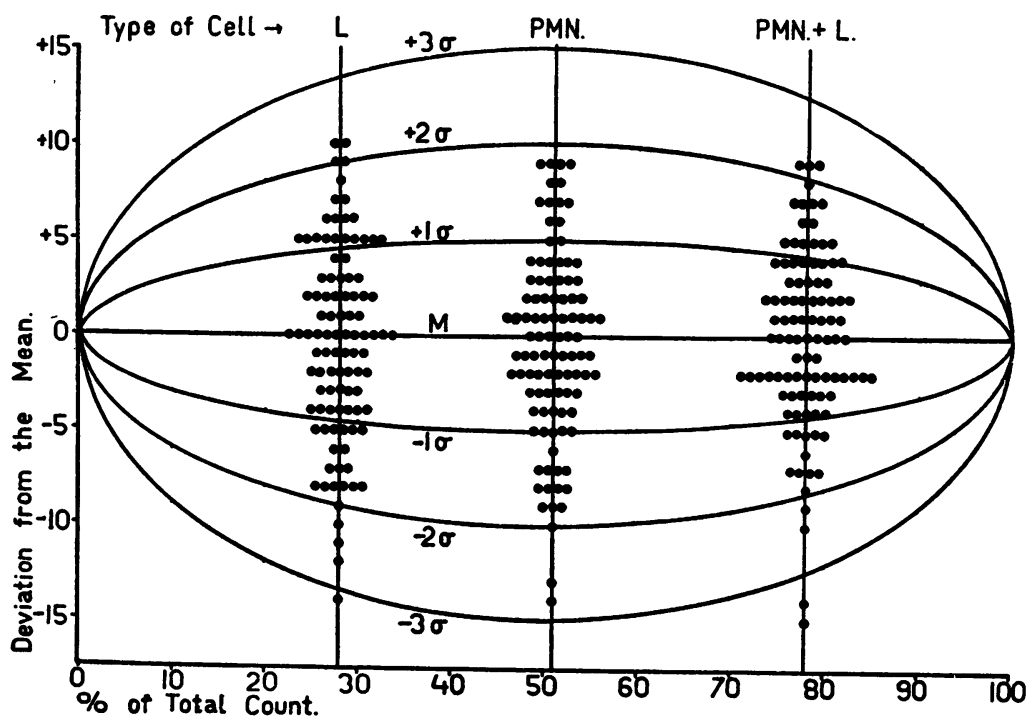

Chart 1. Chart Showing the Deviation of the Individual Counts from the Mean, in Relation to Multiples of the Standard Deviation.

The distribution of observations with relation to the standard deviation is very close to the expected one. If chance alone is responsible for the deviations, and if a large enough number of observations is made, 68.3 per cent of the observations will lie within one standard deviation of the mean, 95.4 per cent within two and 99.7 per cent within three. In the chart it will be seen that three observations of the 300 shown lie outside the line representing three times the standard deviation. This is more than would be expected from chance alone, but again it is probably the result of an added mechanical error.

\section{DISCUSSION}

The results show that the completely unavoidable error in differential blood counts, which is due to chance, and which cannot be eliminated by the most perfect technique, may be of considerable magnitude. It may be decreased by counting larger numbers of cells, and Chart 2 shows the maximum chance errors to be expected with differential counts done on 100,200 , and 400 cells. It is to be noted that the error is inversely proportional not to the number of cells counted but to the square root of the number. In other words, in order to double the accuracy of a count 
it is necessary to count four times the number of cells, and to triple it, nine times.

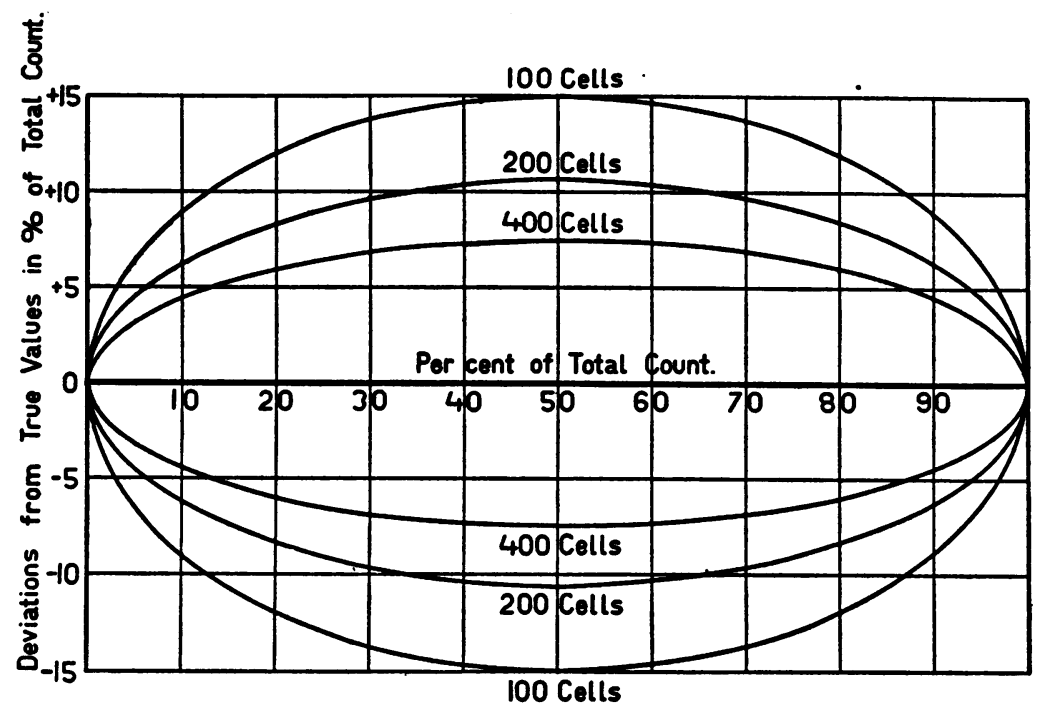

Chart 2. Chart Showing the Maximum Errors Due Only to Chance, in Differential Blood Counts with the Total Counts of 100, 200, and 400 Cells.

Another question which may at times be of considerable importance is that of the significance of a change in the proportion of a certain type of cell in two different counts. Thus, suppose we have a patient suspected of acute appendicitis, whose differential count of 100 cells shows 70 per cent of polymorphonuclears, and an hour later, 80 per cent. Is this a significant change, or may it be due entirely to chance variation? This is decided by determining the standard error of the difference of the two counts according to Yule, page 268. If the observed difference is less than three times the standard error of the difference, it may be entirely the result of chance. The method of calculation is as follows:

$$
\begin{gathered}
p_{0}=\frac{n_{1} p_{1}+n_{2} p_{2}}{n_{1}+n_{2}}, \\
e_{12}^{2}=p_{0} q_{0}\left(1 / n_{1}+1 / n_{2}\right),
\end{gathered}
$$

where $n_{1}$ and $n_{2}$ are the numbers of cells counted in the two counts, $p_{1}$ and $p_{2}$ the proportion of polymorphonuclears in the two counts and $e_{12}$ the standard error of the difference.

In the example just cited, $n_{1}$ and $n_{2}$ each equal 100 and $p_{1}$ and $p_{2}$ are .70 and .80 respectively. Therefore,

$$
\begin{aligned}
p_{0} & =\frac{100 \times .70+100 \times .80}{200}=.75, \\
e_{12}^{2} & =.75 \times .25(1 / 100+1 / 100)=.00375, \\
e_{12} & =.0613 \text { or } 6.13 \text { per cent. }
\end{aligned}
$$


The observed difference of 10 per cent between the percentage of polymorphonuclears in the two counts is about one and a half times this standard error and thus cannot be considered significant.

It is apparent that differential blood counts done on 100 cells, even with the most perfect technique and interpretation, are subject to such wide fluctuations as a result of chance variations in distribution that they are practically without significance. When to this unavoidable error there is added the mechanical error and that of interpretation, the result must necessarily be extremely unreliable. As has been repeatedly pointed out by others, at least 400 cells must be counted before the results of a differential count may be considered at all reliable and even here the maximum chance error may be as much as 7.5 per cent of the total count, as is shown in Chart 2.

To judge the accuracy of a particular count, it is only necessary to read from Chart 2 the deviation to be expected for a certain observed percentage of cells on the curve corresponding to the number of cells counted. This deviation added to and subtracted from the observed value will give an estimate of the range within which the true value will probably lie. Thus if a lymphocyte percentage of $\mathbf{4 0}$ is observed in a count of 200 cells, the vertical line through 40 per cent on the horizontal axis is followed up and down until it intersects the curve marked 200 cells. The points of intersection are at about plus and minus 11 per cent. Consequently the true percentage of lymphocytes may be expected to lie between 29 per cent and 51 per cent.

\section{SUMMARY}

1. The error in differential blood counts due to chance variations in distribution is pointed out, and its magnitude for counts done on various numbers of cells is calculated.

2. One hundred differential counts of 100 cells each on the same blood are reported, and the dispersion of the separate observations is shown to agree closely with the predicted value.

3. It is concluded that at least 400 cells must be counted before the results of a differential count may be considered reliable, and even here the maximum error due entirely to chance is 7.5 per cent of the total count.

\section{BIBLIOGRAPHY}

1. Napier, L. E., Ind. M. Gaz., 1922, lvii, 176. The Differential Blood Count.

2. Meissner, H., Ztschr. f. wissensch. Mikr., 1928, xlv, 280. HämogrammTechnik und Fehlergrenzen der Zählmethoden.

3. Stephens, J. W. W., Yorke, W., Blacklock, B., Macfie, J. W. S., Cooper, C. F., and Carter, H. F., Ann. Trop. Med., 1920, xiv, 371. Have Differential Leucocyte Counts any Value?

4. Beacom, D. N., J. Lab. and Clin. Med., 1928, xiii, 366. Differential Blood Counts; A Comparison of the Accuracy Obtained by Various Methods. 
5. Gyllenswärd, C., Acta Paediat., 1929, viii (Supp. 2), 1. Some Sources of Error at Differential Count of White Corpuscles in Blood-Stained Smears.

6. Dahlberg, G., Acta. med. Scandinav., 1929, lxx, 103. Mittlerer Fehler und Variabilität bei Blutkörperrechnung.

7. Kilgore, E. S., J. A. M. A., 1920, lxxv, 86. Relation of Quantitative Methods to the Advance of Medical Science.

8. Brandt, T., Folia haemat., 1926, xxxii, 177. Über die Fehlerberechnung der hämatologischen Methoden; ein Beitrag zur kritischen Beurteilung der gefundenen Werte.

9. Yule, G. U., An Introduction to the Theory of Statistics. Charles Griffin and Co., Ltd., London, 1922, 6th ed. 